\title{
Retrorectal tumors: report of two cases
}

\section{P Kafle, ${ }^{1}$ Praveen CR, ${ }^{2}$ S Kumar, ${ }^{3}$ BN Patowary, ${ }^{4} \mathrm{~N}$ Maharjan, ${ }^{1} \mathrm{~S}$ Shah,,${ }^{1} \mathrm{~S}$ Agrawal ${ }^{1}$}

${ }^{1}$ Resident Department of Surgery, College of Medical Sciences (COMS) Bharatpur, Nepal. ${ }^{2}$ Lecturer General Surgery. ${ }^{3} \mathrm{MCh}$ Resident Surgical Gastroenterology. ${ }^{4}$ Prof \& Head, Department of Gastrosurgery.

\begin{abstract}
Retrorectal tumor is uncommon identity presenting with nonspecific sign and symptoms making difficulty in diagnosis. Benign tumors are more common than malignant. The lesion may be malignant or progress to malignancy from benign state. Retrorectal masses in young women may continue to grow and result in dystocia. Cystic lesions are also at risk of becoming infected, which renders subsequent excision more difficult and increase the risk of recurrence. Cross-sectional imaging is required to determine the extent of resection and the appropriate surgical approach. Surgical removal leads to favorable outcomes for patients with benign purely cystic retrorectal tumors. We report two such benign rectal lesions.
\end{abstract}

Key words: Retrorectal tumors, Presacral space, Mass.

\section{Introduction}

Primary tumors of the retrorectal (or presacral) space are rare lesions generally originating from the embryologic remnants of this space. ${ }^{1,2}$ Retrorectal space is bounded anteriorly by fascia propria, posteriorly by presacral fascia overlying the sacrum, the peritoneal reflection superiorly, Waldeyer's fascia inferiorly, and the lateral stalks of the rectum, the ureters and the iliac vessels. ${ }^{2}$ The incidence is 1 in 40,000 hospital admissions in a large series from the Mayo Clinic. The majority of retrorectal tumors are benign. These tumors are broadly categorized as inflammatory, congenital, neurogenic, osseous, and miscellaneous. ${ }^{1}$ In general two-thirds are congenital in origin, of which a further two-thirds are developmental cysts, and the next most common masses are neurogenic tumors. Developmental cysts may originate from any of the three

Correspondence: Dr. Prakash Kafle

Email:prakashkaflee@hotmail.com germ layers and include epidermoid and dermoid cysts, enterogenous cysts, tailgut cysts, and teratomas. ${ }^{3}$ Epidermoid and dermoid cysts are the direct result of closure failure of the ectodermal tube. Epidermoid cysts are composed of stratified squamous cells and are typically benign unilocular lesions that do not contain skin appendages. Unlike epidermoid cysts, dermoid cysts have stratified squamous epithelium with skin appendages (sweat glands, hair follicles, sebaceous cysts). Both type of cysts may communicate with the skin and be associated with a postanal dimple or sinus. Teratomas are true neoplasms. They include all three germ layers; epithelium of the gastrointestinal tract, respiratory tract, and nervous system. These lesions can be solid or cystic and often contain both components. If germ cell components are present, teratomas have the potential to undergo malignant degeneration to squamous cell carcinoma or 
P Kafle et al. Retrorectal tumors: report of two cases

rhabdomyosarcoma. Teratomas are more common in female compared with male. In the adult population there is a risk of 40 to $50 \%$ malignant degeneration and which increases with incomplete resection. These lesions tend to adhere to the coccyx and surgical approach requires en bloc coccygectomy. ${ }^{4}$ We report two such benign cases, found in females. Retrorectal tumors are frequently asymptomatic, and they are often found incidentally. Patients may initially complain of constipation, paradoxical diarrhea, or frequent urge to defecate. In our cases they had difficulty in defecation and often painful. Even symptomatic patients may be misdiagnosed as having fistulae in ano, pilonidal cysts, perianal abscesses, psychogenic, post-traumatic, or postpartum pain, or proctalgia fugax. Retrorectal tumors report are almost always palpable on digital rectal examination (DRE). Both of our cases had rectal mass on posterior wall. The presence of a postanal skin dimple is suggestive of a developmental cyst. ${ }^{1}$ In one of our cases there was postanal dimple.

\section{Case Details}

Case 1: 37 years aged female presented with complaint of low back pain, low mid line mass near to anus and difficulty during defecation, with no neurological symptoms in the lower extremities. On evaluation 3x4 cm nodular mass over lower midline 3 $\mathrm{cm}$ away from the anal verge (Fig 1). On digital rectal examination a firm mass was palpable against the posterior wall of the rectum, with no involvement of mucosa. Colonoscopy revealed normal findings. Computed tomography(CT) scan revealed localiesd well circumscribed mass in the posterior to rectum with no evidence of sacral or rectal wall involvement (Fig 1). Diagnosis of benign retrorectal tumor was made and total excision along with coccyx via the posterior approach and primary closure of wound was performed. Histopathology confirmed sacrococcygeal teratoma. Postoperative period was uneventful and the patient discharged on $5^{\text {th }}$ postoperative day. On follow up at 6 months she didn't have painful defecation and there was no recurrence

Figure 1: sacrococcygeal teratoma (Arrow)
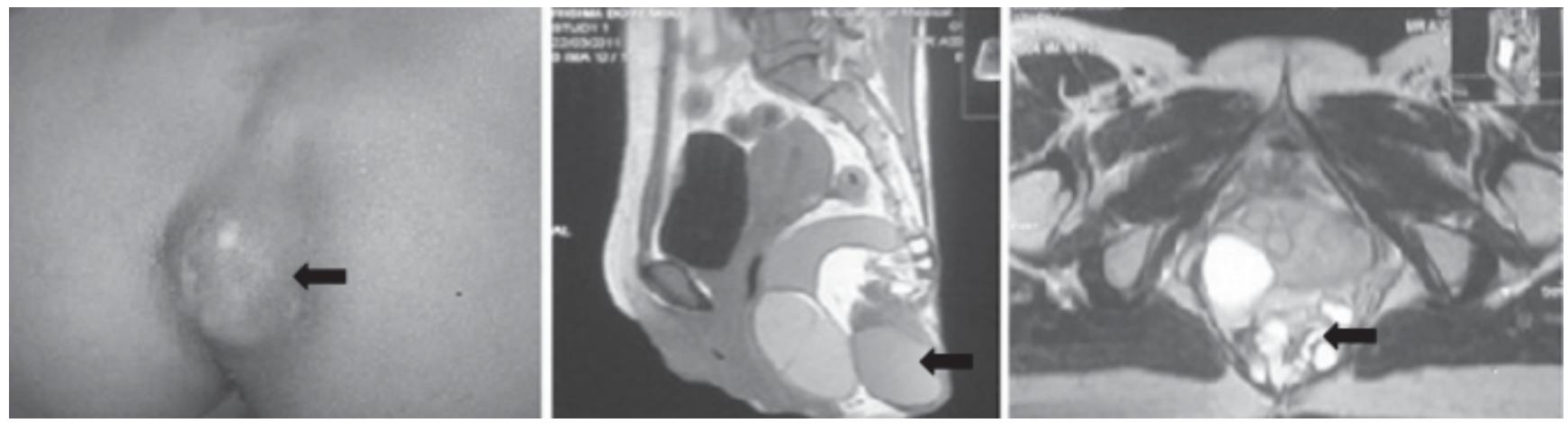

Case 2: 27 years aged female presented with complaint of difficulty in defecation for 8 years with no history of perineal discharge and per rectal bleeding. On evaluation there was a low midline dimple near the anal verge with no external opening. On digital rectal examination, a soft mass felt against posterior wall of the rectum with no involvement of the mucosa. Colonoscopy was not remarkable. CT imaging revealed features suggestive of benign retrocaecal mass on the retrorectal space as shown in the Figure 2. 
Diagnosis of benign lesion was made and posted for surgical excision. Total excision of the mass along with coccyx was done through posterior approach. Histopathology confirmed it as dermoid cyst. Patient had wound infection and was allowed to heal by secondary intention. On her follow up, at 4 months she was symptom free and no recurrence.

\section{Figure 2: dermoid cyst(arrow)}
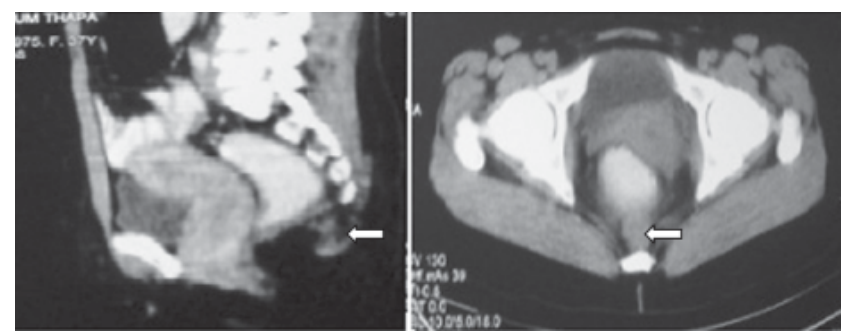

Figure 3: Gross specimen of (a) Sacrococcygeal teratoma \& (b) Dermoid cyst

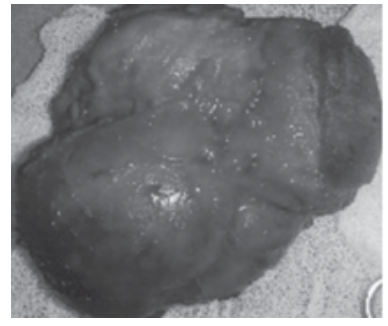

$\mathrm{a}$

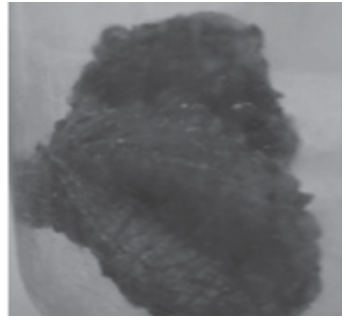

b

\section{Discussion}

The Retrorectal tumors are extremely rare. True incidence is difficult to estimate. Though retrorectal tumors are scarce, they should be taken into consideration for those who present with a posterior mass on rectal examination. Adult retrorectal tumors mainly occurred in female, aged 40 to 60years, while malignant ones developed frequently in males. ${ }^{5}$ Retrorectal tumors can be completely asymptomatic throughout their course. The presenting symptoms are usually related to compression of or invasion to the surrounding pelvis viscera and nerve. Urologic symptoms, change of bowel habits, and nerve root signs are common. The differential diagnosis of masses within this space is broad like neurogenic, osteogenic and congenital origin, metastatic and inflammatory processes. Congenital lesions include chordomas, teratomas, anterior sacral meningoceles, and developmental cysts (dermoid, epidermoid, enteric duplication, and tailgut cysts). Excluding inflammatory lesions, developmental cysts are the most common masses in the retrorectal space. ${ }^{6}$ Colonoscopy can examine the rectal lining and delineate the proximal extent of the tumors. CT and MRI provide excellent direct anatomical description regarding the lesions. ${ }^{5} \mathrm{CT}$ imaging distinguishes whether tumors are cystic, solid or mixed (solid and cystic components). Moreover, CT imaging determines if other pelvic structures such as the bladder, uterus, ureters, or rectum are involved. Cortical bone destruction is also demonstrated by CT, whereas MRI is superior in evaluation of marrow involvement. MRI, by improving soft tissue resolution, is quite helpful in planning the extent of resection of adjacent structures. MR angiogram or venogram may add additional information regarding vascular involvement and indicate theneed for a vascular surgeon to be a member of the multidisciplinary surgical team. In our cases these investigations were not carried out as both cases were benign and with no neurological or bone involvement. Endoanal ultrasound may be helpful in determining the relationship of a retrorectal mass to the wall of the rectum. Preoperative tissue biopsy is controversial. ${ }^{6}$ Some have stated that all solid or mixed solid/cystic tumors should be biopsied prior to surgical intervention. ${ }^{7-9}$ In both our cases prior biopsy was not performed. If tissue diagnosis of a retrorectal mass is required, a needle biopsy should be performed by an 
P Kafle et al. Retrorectal tumors: report of two cases experienced radiologist and within the field of the proposed area of resection so that the needle tract may be excised en bloc with the specimen at the time of operation if turns out to be malignant on histopathological report.

All the tumors in this space should be excised completely. Anterior, posterior, and combined approaches are alternative techniques depending on the size, location, and spatial relationship of the tumors. For small $(<1 \mathrm{~cm})$ and low-lying tumors, within the level of sacral promontory, posterior approach is favored. Anterior approach is preferred for larger, highlying, or high-grade malignant tumors because it provides better visualization of pelvic structures, bleeding control, and easier mobilization of rectum. Iatrogenic surgical damage to surrounding structures includes urine/fecal incontinence, nerve root trauma, structural defect, and abscess/fistula formation. ${ }^{5}$ In our series we did complete excision through posterior approach which on histopathological examination were confirmed to be benign lesions i.e. sacro-coccygeal teratoma \& dermoid cyst.

\section{Conclusion}

Retrorectal space tumors are uncommon lesions that present with nonspecific signs and symptoms. A high index of clinical suspicion is required for diagnosis. Cross sectional imaging is mandatory for evaluation of retrorectal tumors, and it assists in planning the correct surgical approach by determining the extent of the tumor and nature of the lesion. Preoperative tissue diagnosis with image-guided biopsy is considered essential for the management of solid and heterogeneously cystic tumors. Purely cystic lesions should not be biopsied unless there is a high suspicion of malignancy. These tumors must be managed with a multidisciplinary approach to maximize successful outcome thus reducing the postoperative morbidity.

\section{References}

1. Sean C. Glasgow and David W. Dietz. Retrorectal Tumors. Clin Colon Rectal Surg 2006;19:61-8.

2. B. Bulent Mentes, Osman Kurukahvecioglu, Bahadýr Ege, et al. Retrorectal tumors: A case series. Turk J Gastroenterol 2008;19:40-4.

3. Jao SW, Beart Jr RW, Spencer RJ, et al: Retrorectal tumors: Mayo Clinic experience, 1960-1979. Dis Colon Rectum 1985;28:644.

4. Jeffrey A. Neale. Retrorectal Tumors. Clin Colon Rectal Surg 2011;24:149-60.

5. Hao-Cheng Chang, Po-Fang Wang, Jy-Ming Chiang, et al. Outcomes of Surgical Intervention for Retrorectal Tumor: Experience of Sixteen Patients in Chang Gung Memorial Hospital. J Soc Colon Rectal Surgeon 2010;21:87-94.

6. Killingsworth C, Gadacz TR. Tailgut Cyst (Retrorectal Cystic Hamartoma): Report of a Case and Review of the Literature. Am Surg. 2005;71:666-73.

7. Lev-Chelouche D, Gutman M, Goldman G, et al: Presacral tumors: A practical classification and treatment of a unique and heterogeneous group of diseases. Surgery 2003;133:473.

8. Milsom JW, Fazio VW, et al: Our approach to the management of congenital presacral tumors in adults. Int J Colorectal Dis 1993;8:134. 
Journal of College of Medical Sciences-Nepal, 2012, Vol-8, No-4

9. Eilber FR: Expert commentary on Dozois RR. Retrorectal tumors: Spectrum of disease, diagnosis and surgical management. Perspect Colon Rectal Surg 1990;3:241.
10. Hughes DE, Lamb J, Salter DM, et al: Fineneedle aspiration cytology in a case of chordoma. Cytpoathology 1992;3:129.

11. Mathis KL, Dozois EJ, Grewal MS, et al: Malignant risk and surgical outcomes in presacral tailgut cysts. Br J Surg 2010;97:575. 